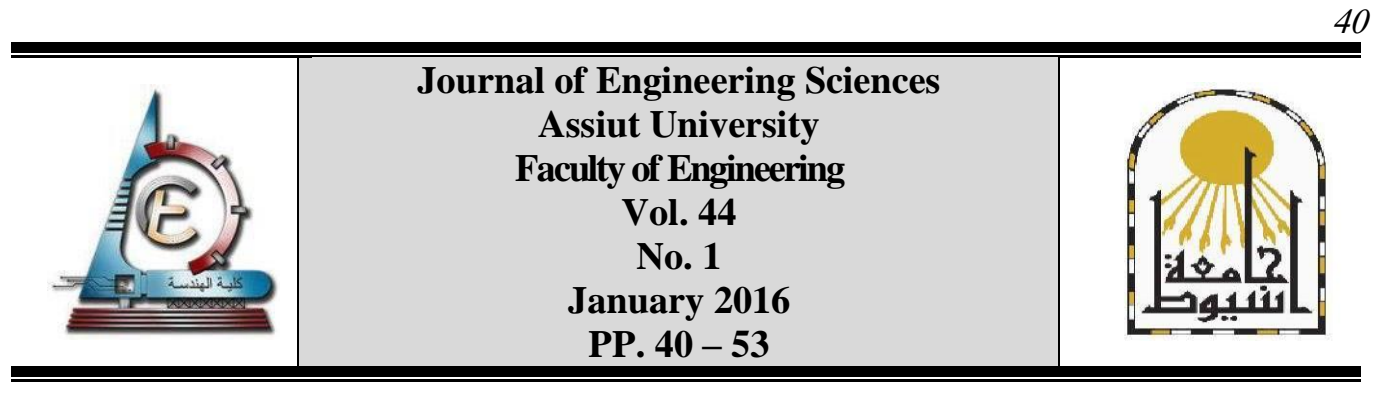

\title{
A SPATIALLY QUANTIZED POWER AND RESOURCE ASSIGNMENTS FOR CELLULAR \& D2D COMMUNICATIONS
}

\author{
Gamal A. F. M. Khalaf and Hesham. M. Z. Badr \\ Communications Dep., Faculty of Engineering, Helwan, Cairo, Egypt
}

Received 20 October 2015; Accepted 20 November 2015

\begin{abstract}
This work presents a tractable approach to enabling D2D uplink underlay in cellular networks in order to improve the spatial frequency reuse and to enhance the performance of cellular networks. In general, the cellular-to-D2D interference needs to be restricted to maintain a target performance level of the networks. In this respect, we propose an optimally quantized transmission power control and interpolation schemes for cellular users to limit the interference from them on D2D users. On the other hand, for the interference induced from D2D transmissions on the base station's receiver a concentric cell structure in conjunction with an orthogonal resource assignment scheme is developed. The proposed schemes exploit the assistance of a localization system developed to estimate the D2D and cellular user's spatial separations. Simulation results show that the proposed schemes can bring less interference and, at the same time, can easily be implemented in real cellular networks without the need of further standardization work.
\end{abstract}

Keywords: D2D integration, optimal quantized power assignment, resource allocation, concentric cell structure, Linear programming.

\section{Introduction}

Device-to-device (D2D) communications in cellular spectrum supported by cellular infrastructure has the potential of increasing spectrum and energy efficiencies. This is made possible by taking advantage of the so called proximity and reuse gains [1] - [5]. Integrating D2D in LTE-Advanced network is a research topic that attracts many industrial interests and is being rapidly developed in the 3GPP LTE standardization (3GPP) to facilitate proximity aware internetworking services [6]. Services and applications based on D2D communications include, as a motivating example, national security, and public safety applications [7] and machine type communications [8].

The main idea in D2D-enabled cellular networks is to permit transmitter-receiver pairs coexisting in close proximity to establish direct connections between each other. That is, if a transmitter has its designated receiver within a "proximity" range, the transmitter is

* Corresponding author.

Email address:gam_hel@yahoo.com 
JES, Assiut University, Faculty of Engineering, Vol. 44, No. 1, January 2016, pp. 40 - 53

allowed to bypass the base station (BS) and communicate in a D2D direct mode. Therefore, D2D communication enables short-range, low-power links to coexist with the cellular links and thereby improves the spatial reuse of the available spectrum, decrease the (transmission) power consumption in the user equipments (UEs) and improves the total network throughput by offloading some of base station's traffic [9]-[12].

In order to realize D2D communication, a number of important procedures need to take place [6]. This includes, i) Mode Selection (MS): for a D2D candidate pair consisting of a D2D transmitter and receiver that are in proximity. The MS algorithm needs to decide on one of 3-possible communication modes: cellular mode, D2D direct mode with dedicated resources or D2D mode with reused resources. This latter case involves, ii) Resource Allocation (RA): in order to decide on which D2D pair(s) is sharing resources with which cellular UE [7]. In LTE, the basic resource unit of the OFDM (orthogonal frequency division multiplexing) system consists of 12 continuous subcarriers for a duration of $1 \mathrm{~ms}$ (one slot), is termed a Resource Block (RB) which is the smallest unit of resource that can be allocated to one UE. A D2D enabled UE can reuse some of the OFDM time-frequency physical RBs.

D2D communications utilizing cellular spectrum poses new challenges, because the network needs to cope with new interference situations. For example, in an OFDM system, due to the reuse, intra cell orthogonality could be lost and, hence, interference can become severe due to the random positions of the D2D transmitters (receivers) and of the cellular UEs communicating with their serving BS [8], [10]. Apart from mode selection and resource allocation, iii) Power Control (PC) is a key technique which can deal with intraand intercell interferences [11] - [14]. As the previous procedures in D2D communications, iv) D2D Discovery is another key step to determine which user(s) is a potential D2D UE based on both the D2D link distance, $r_{d}$, and the cellular link distance, $r_{c}$ (depicted in Fig.(1) below). Before D2D communications between any two UEs can happen, the network must estimate the spatial separations of the potential D2D users as well as that of the cellular user's. While the positions of the D2D candidates and the cellular users can be obtained from GPS (Global Position System) or A-GPS (Assisted GPS). This approach does not suite the LTE D2D because GPS signals have poor penetration through walls. For the purpose of this paper, a simple, inexpensive scheme to estimate the spatial separations between UEs based on acquired received signal strength (RSS) indications is presented.

The paper is organized as follows. In Section 2, a D2D and cellular user's coordinate estimation system is presented. This is followed by a detailed optimum power assignment and interpolation scheme in section 3. Section 4 is devoted to describing a spatial resource allocation system based on concentric cell structure. Section 5 focuses on the outage probability as our metric chosen for network performance evaluation. Simulation and validation results are presented in section 6. Section 7 summarizes the research work carried out in this paper and conclusions are drawn. 


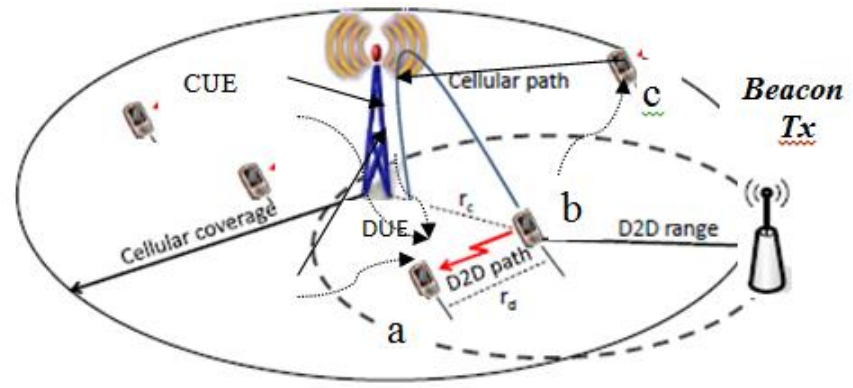

Fig. 1. D2D enabled cellular network [20].

\section{D2d spatial coordinate estimation}

In this section, a simple, practical approach for UE's spatial separation estimation in a typical cellular network scenario is proposed. The aim is to enable efficient power and resource assignments between proximate D2D users. Consider the "coverage extension" scenario wherein a UE can obtain access to the LTE cellular network through assistance of a multi-hop relay [15], [17]. In this paper, we propose to extend the network coverage extension function of the multi-hop relay to include participating in the coordinate estimation of UEs at low cost and complexity. Assume that a relay is being placed at the border of a cell with known, fixed $(X, Y)$ coordinates (i.e., the beacon Tx on the right of Fig. (1)). Assume, also, that the relay is reliably, continuously transmitting a beacon signal marking its $I D$ and its (fixed) coordinates. Hence, the mean signal strength (RSS) received at a given UE is given by, [18],

$$
\overline{\mathrm{P}}_{\mathrm{ij}}\left(\mathrm{dB}_{\mathrm{m}}\right)=\Pi_{0}\left(\mathrm{~dB}_{\mathrm{m}}\right)-10 \mathrm{n}_{\mathrm{p}} \log _{10}\left(\mathrm{~d}_{\mathrm{ij}} / \Delta_{0}\right)
$$

Where, $\bar{P}_{i j}\left(\mathrm{~dB}_{\mathrm{m}}\right)$ is the mean received power (in $\left.\mathrm{dB}_{\mathrm{m}}\right)$ at $\mathrm{UE} j$, transmitted by the relay device $i, \Pi_{0}$ is the received power at a reference distance $\Delta_{0}$ (typically $=1 \mathrm{~m}$ ), is calculated from free space formula [16], $n_{p}$ is the path loss exponent (2:6) and $d_{i j}$ is the transmission path-length (range) between the UE and the relay device. From Equ., (1), the communication range $d_{i j}$ is given by,

$$
\mathrm{d}_{\mathrm{ij}}=\Delta_{0} 10 \frac{\Pi_{0}(\mathrm{dBm})-\overline{\mathrm{P}}_{\mathrm{ij}}(\mathrm{dBm})}{10 \mathrm{n}_{\mathrm{p}}}
$$

Now, consider a two-dimension rectangular area with length $X_{a}$ and width $Y_{a}$. In this area, the BS is located at the origin, $(X=0, Y=0)$, while the multi-hop relay is at position $\left(X=X_{a}, Y=0\right.$ (Fig. (1)). For any $\mathrm{UE} i$, in the area with coordinates $(X(i), Y(i))$, the following equations hold [18],

$$
\begin{gathered}
\mathrm{X}^{2}(\mathrm{i})+\mathrm{Y}^{2}(\mathrm{i})=\mathrm{d}_{1}^{2}(\mathrm{i}) \\
\left(\mathrm{X}(\mathrm{i})-\mathrm{X}_{\mathrm{a}}\right)^{2}+\mathrm{Y}^{2}(\mathrm{i})=\mathrm{d}_{2}^{2}(\mathrm{i}) \\
0 \leq \mathrm{X}(\mathrm{i}) \leq \mathrm{X}_{\mathrm{a}} \\
0 \leq \mathrm{Y}(\mathrm{i}) \leq \mathrm{Y}_{\mathrm{a}}
\end{gathered}
$$


Where, $\mathrm{d}_{1}(\mathrm{i})$ is the distance between UE $i$ and the BS and $\mathrm{d}_{2}$ (i) is that between UE $i$ and the relay device. Solving Equs. (3), (4) gives the UE's coordinates,

$$
\begin{array}{r}
\mathrm{X}(\mathrm{i})=\frac{d_{1}^{2}(\mathrm{i})-d_{2}^{2}(\mathrm{i})+X_{a}^{2}}{2 \mathrm{X}_{\mathrm{a}}}, \\
\mathrm{Y}(\mathrm{i})=\sqrt{\mathrm{d}_{1}^{2}(\mathrm{i})-\mathrm{X}^{2}(\mathrm{i})}
\end{array}
$$

As can be seen, using Eqs., (2)-(6), each UE can compute and, hence, update its $(X, Y)$ coordinate to the BS. That is, a given UE measures the RSS of the BS's transmission and that of the beacon signal sent by the relay device, then, it can determine its $(X, Y)$ coordinate and report back its coordinate to the BS. Consider, for instance, the Mode Selection scheme wherein the BS needs to decide on one of possible communication modes (cellular or D2D) for the 3-UEs, $a, b, c$, shown in Fig., (1) above. The BS can calculate the distance between UEs, $a$ and $b$ as follows,

$$
d_{a, b}=\sqrt{\left(X_{b}-X_{a}\right)^{2}+\left(Y_{b}-Y_{a}\right)^{2}},
$$

And, that between the $a-b$ pair and UE $c$, as follows,

$$
d_{a b, c}=\sqrt{\left(X_{c}-\frac{X_{a}+X_{b}}{2}\right)^{2}+\left(Y_{c}-\frac{Y_{a}+Y_{b}}{2}\right)^{2}}
$$

Given the above spatial separations and based on the state of the cellular links at the $\mathrm{BS}$, it can select the proper communication mode (explained later). An example on spatial separation between UEs estimation is depicted in Fig., (2) below.

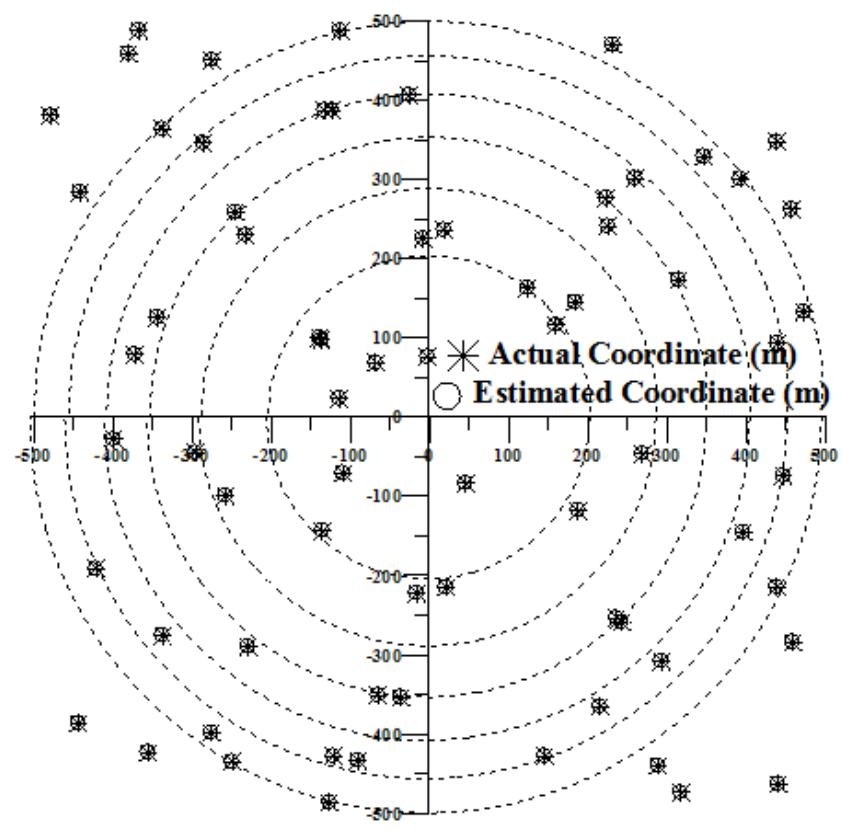

Fig. 2. UE's coordinates estimation in a concentric cell.

\section{Even-odd spatial resource allocation}

With the aid of the UE's localization scheme presented in section 2, we propose a spatial, orthogonal resource reuse scheme which can guarantee an average SINR level for both the cellular and the D2D underlay. 
Without loss of generality, assume uniform user distribution over all the cell area, and that the whole cell area is divided into non overlapping (soft) concentric subareas as depicted in Fig. 2. Now, let each subarea be assigned a set of resource blocks (RBs) enough to maintain a target level of performance in the cellular network. At the same time, each subarea is divided into two spatially disjoint zones: a-zones (upper half of the cell) and $b$-zones (lower half of the cell) as depicted in Fig., (3) below. In order to distinguish different $a, b$ zones, we use the $(N, a)$ convention to signify the $a$-zone of subarea $N$. Now, let each RB set be divided into disjoined odd-subset used in the a-zones and even-subset used in the $b$-zones. Therefore, the same odd-subset of RBs in a given subarea $k:(k, a)$, (e.g., zone $c$ in Fig., (3)) can be reused in zone $(N-k+1, b)$, (e.g., zone $d$ ). This arrangement provides sufficient spatial separations between simultaneous cellular and D2D transmissions. This, in turn, should guarantee a minimum interference levels induced at the D2D and at the cellular receivers. An example on a concentric cell with equal subareas and its associated RB sets/subsets assignment are shown in Fig., (3) and its geometry are summarized in Table (I) for equal subareas as a special case.

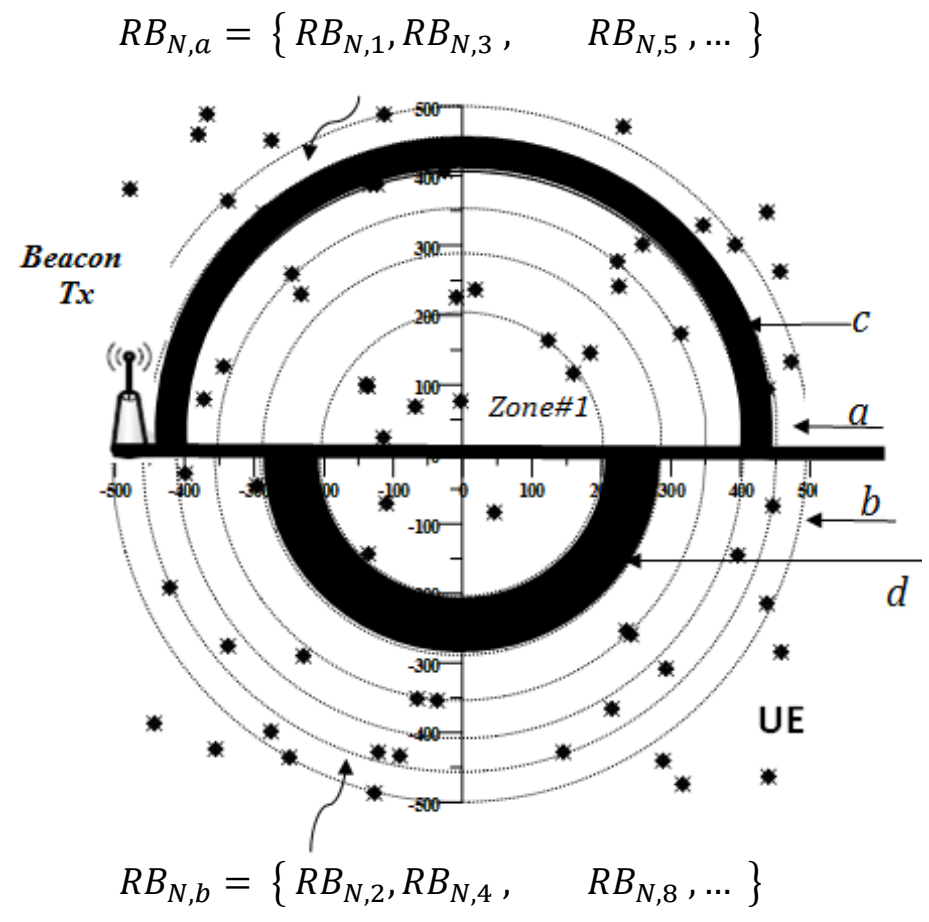

Fig. 3. N-concentric cell with equal subareas.

Table 1.

Radii of equal subareas

\begin{tabular}{|c|c|c|c|c|c|}
\hline $\begin{array}{c}\mathrm{R}_{0,} \\
\text { cell radius }\end{array}$ & $\mathrm{R}_{1}$ & $\mathrm{R}_{2}$ & $\mathrm{R}_{3}$ & $\mathrm{R}_{4}$ & $\mathrm{R}_{5}$ \\
\hline $500 \mathrm{~m}$ & $\approx 456 \mathrm{~m}$ & $\approx 408 \mathrm{~m}$ & $\approx 353 \mathrm{~m}$ & $\approx 288 \mathrm{~m}$ & $\approx 204 \mathrm{~m}$ \\
\hline
\end{tabular}

As can be seen, odd-RB subset is allocated to zone $a$ of subarea $k,(k, a): \operatorname{RB}_{\mathrm{k}, \mathrm{a}}=$ $\left\{\mathrm{RB}_{\mathrm{k}, 1}, \mathrm{RB}_{\mathrm{k}, 3}, \mathrm{RB}_{\mathrm{k}, 5}, \ldots\right\}$ while even-RBs subset is allocated to zone $b$, of same subarea 
JES, Assiut University, Faculty of Engineering, Vol. 44, No. 1, January 2016, pp. 40 - 53

$(k, b): \mathrm{RB}_{\mathrm{k}, \mathrm{b}}=\left\{\mathrm{RB}_{\mathrm{k}, 2}, \mathrm{RB}_{\mathrm{k}, 4}, \mathrm{RB}_{\mathrm{k}, 6}, \ldots\right\}$. At the same time, odd $\mathrm{RBs}$ of zones $(k, a), k=$ $1,2 \ldots N$, can be reused in zones $(N-k+1, b)$. This subset/zone layout and its associated RB assignments are assumed to be kept fixed and do not change over time. Moreover, we assume that the RB assignment-data are stored in the memory of the BS. At this point, it is not difficult to realize the following:

1) By exploiting the coordinate estimation scheme presented in Section 2, the BS can select the potential D2D users and the corresponding $R B(s)$ to be reused such that a given SINR performance threshold, $\varphi$, is guaranteed.

2) Extending the above subarea/zone layout and its associated $R B$ set/subset assignments into sectored cell cases is straightforward.

3) Once again, the resource allocation technique being proposed can be employed in any existing cellular system without the need of a new standardization work.

Finally, this set/subset assignment scheme has shown a promising performance when compared with the state-of-the-art (conventional non-concentric) assignment algorithms as will be seen in subsequent section.

\section{Spatially quantized optimum transmission power assignment}

In this section, we propose an optimum transmission power control system employing, i) discrete optimum transmission power assignment to set the UE's transmission power so that (based on the spatial separations between the UEs) a given power level is used enough to compensate for the channel path loss as well as for the estimated interference and noise power, ii) power interpolation scheme that extends the discrete power assignments so that it covers all the cell area.

\subsection{Optimum discrete transmission power assignment}

In this framework, the proposed power control system is based on finite set of optimal transmission power levels. Specifically, As mentioned earlier, assume a cell consisting of $N$ concentric [18], whose radii are, $R_{0}, R_{1}, R_{2}, \ldots, R_{N-1}$ with decreasing lengths with $R_{0}$ being the cell radius as depicted in Fig., (3). Here, the area of each subarea is identified by selecting two adjacent radius values, say, $R_{i}$ and $R_{i+1}$, for the $i t h$ subarea. Let us assume that, at the boarder of each subarea $k$, UEs use transmission power level, $P_{T}^{k}, k=1,2, \ldots$ $N$. This assumption is relaxed in the subsequent. We consider path-loss attenuation and the effect of exponentially distributed Rayleigh fading, $\mathrm{h}_{i}^{k}$, from the serving station $i$. In particular, if the transmitted power in the border of subarea $k$ is $P_{T}^{k}$ and the path-loss exponent is $\alpha>2$, then the received power, $P_{R}^{i}$ at a receiver $i$ is given by [7],

$$
\mathrm{P}_{\mathrm{R}}^{\mathrm{i}}=\mathrm{P}_{\mathrm{T}}^{\mathrm{k}} \mathrm{h}_{\mathrm{i}}^{\mathrm{k}} \mathrm{d}_{\mathrm{k}, \mathrm{i}}^{-\alpha} \text {, }
$$

and the SINR (signal to interference and noise) experienced at the intended ( cellular or $\mathrm{D} 2 \mathrm{D})$ receiver is given by,

$$
\operatorname{SINR}=\frac{\mathrm{P}_{\mathrm{T}}^{\mathrm{k}} \mathrm{h}_{\mathrm{i}}^{\mathrm{k}} \mathrm{d}_{\mathrm{k}, \mathrm{i}}^{-\alpha}}{\sigma^{2}+\sum_{\mathrm{n}=1}^{\mathrm{N}} \sum_{\forall \mathrm{j} \neq \mathrm{k}} \mathrm{P}_{\mathrm{T}}^{\mathrm{j}} \mathrm{h}_{\mathrm{i}}^{\mathrm{j}} \mathrm{d}_{\mathrm{j}, \mathrm{i}}^{-\alpha}} \geq \varphi
$$

Where, $\mathrm{n}$ signifies the concentric subarea, $1,2 \ldots \mathrm{N}, d_{j, i}>1$ is the distance between transmitter $j$ and receiver $i$ and $\varphi$ designates (equal) minimum SINR requirement either for D2D or cellular users. Here, $\sum_{n=1}^{N} \sum_{\forall j \neq k} \mathrm{P}_{T}^{\mathrm{j}} \mathrm{h}_{\mathrm{i}}^{\mathrm{j}} \mathrm{d}_{\mathrm{j}, \mathrm{i}}^{-\alpha}, j=1,2 \ldots$, represents the overall 
interference power at a given receiver $i, \sigma^{2}$ is the mean noise power. In the following, we use simple example to derive a system of equations such that an optimal (boarder) transmission-power assignment is formulated as a linear programming problem. Consider the cell which is being partitioned into $N$ subareas with a single UE located at the border of each subarea (Fig., (3)). Assuming unit mean channel fading, the SINR experienced at the associated receiver(s) satisfies the following Equation(s),

$$
\mathrm{P}_{\mathrm{T}}^{\mathrm{k}} \mathrm{d}_{\mathrm{k}, \mathrm{i}}^{-\alpha}-\varphi \sum_{\mathrm{n}=1}^{\mathrm{N}} \sum_{\forall \mathrm{j} \neq \mathrm{k}} \mathrm{P}_{\mathrm{T}}^{\mathrm{j}} \mathrm{d}_{\mathrm{j}, \mathrm{i}}^{-\alpha} \geq \varphi \sigma^{2}, \quad \mathrm{k} \neq i,
$$

If the distances $d_{k, i}, \forall i, k \neq i$, the noise power $\sigma^{2}$ are known as well as the minimum SINR threshold, $\varphi$, requirements, equations (12) can be solved as a linear programming optimization problem:

$$
\text { Minimize } \sum_{k} \mathrm{P}_{\mathrm{T}}^{\mathrm{k}}
$$

$$
\text { s.t, } \quad \mathrm{P}_{\mathrm{T}}^{\mathrm{k}} \mathrm{d}_{\mathrm{k}, \mathrm{i}}^{-\alpha}-\varphi \sum_{\mathrm{n}=1}^{\mathrm{N}} \sum_{\forall \mathrm{j} \neq \mathrm{k}} \mathrm{P}_{\mathrm{T}}^{\mathrm{j}} \mathrm{d}_{\mathrm{j}, \mathrm{i}}^{-\alpha} \geq \varphi \sigma^{2}
$$

The formulation presented by (13) can easily be employed in any existing cellular network without the need of new standardization work. That is, the BS can solve the optimization problem (13) off line and stores the optimal (border) transmission power levels corresponding to each spatial subarea in a lookup table. This table can be broadcasted by the BS on a less frequent basis. Therefore, a given UE which measures the RSS of the beacon sent by the BS (in LTE, the common reference signal plays the role of the beacon signal) and that sent by the multi-hop repeater (section 2 ). It can then, calculates its $(X, Y)$ coordinate (i.e., Equs. (5),(6)). Therefore, it can, automatically, interpolates its optimal transmission power (section 4.3) level based on the subarea in which it is currently resides. Moreover, if the UE moves to different spatial subarea, it can exploit the new $R S S$ to identify the new power level. Our approach reduces the need of extra channel state knowledge at the BS, and no major modifications to the standard systems. This can be explained as follows. In LTE, the common reference signal (CRS) plays the role of the beacon signal. Therefore, UE's position and other measurement needed by the base station in order to implement our approach can, in principle, be implemented in the similar way by dividing the subcarriers into more parts. Such a modification could be envisioned as, relatively, minor (S/W) modifications to the schemes already realized by all cellular systems when supporting the handover function.

Of importance here is to note that the transmission power minimization problem (13) is the dual of the transmission rate maximization problem. This duality means that (13), will have the same solution in terms of the per user power values when the minimum power sum is used to achieve the maximum (Shannon) transmission rate sum.

\subsection{User's mode selection}

In effect, mode selection plays a similar role for D2D communications as handover does for traditional cellular communications in the sense that the D2D transmitter can switch its transmission between the D2D receiver and its serving base station (BS). Specifically, the BS decides on a potential D2D mode if the D2D link (possibly biased) quality is better than that of the cellular uplink. As such, it is not difficult to realize, from Equ. (12), that the mode selection scheme being adopted here is a distance-based mode selection utilizing the localization scheme proposed in Section 2. 


\subsection{Optimal-transmission power interpolation}

For UEs which do not reside on the subarea's border, they use the optimal "border" power levels (broadcasted as a lookup table by BS) in computing their transmission power at any $(X, Y)$ position in the cell. More specifically, we assume that the border's power levels lie on an underlying but unknown curve and the problem is to interpolate the values of the curve at any $(X, Y)$ position between the borders. In this respect, we use the Hermite interpolation method which is similar to the cubic method (requires at least 4 points) [19], but achieves higher degree of continuity. Results of the UE's power level interpolations are presented in subsequent section. It is not difficult to realize that, the power assignment being proposed in this section reduces the complexity of exhaustively searching for the optimal power allocation for each UE especially for large networks. Moreover, the power interpolation scheme has actually turned the discrete (quantized) power assignment into a continuous assignment scheme overall the cell area.

\subsection{The D2D optimal transmission power assignment}

Our aim is to restrict the D2D interference power induced at the BS by controlling the transmission power of the D2D UEs. In this respect, assume a given SINR target, $\varphi_{\mathrm{D}}$, for the D2D UEs. According to Equ. (11), the D2D transmission power can be calculated as follows:

$$
\mathrm{P}_{\mathrm{T}}^{\mathrm{D}}=\frac{\varphi_{\mathrm{D}}}{\mathrm{d}_{\mathrm{k}, \mathrm{i}}^{-\alpha}}\left(\sigma^{2}+\sum_{\mathrm{n}=1}^{\mathrm{N}} \sum_{\forall \mathrm{j} \neq \mathrm{k}} \mathrm{P}_{\mathrm{T}}^{\mathrm{j}} \mathrm{d}_{\mathrm{j}, \mathrm{i}}^{-\alpha}\right)
$$

Where, $d_{k, i}$ designates the spatial distance between the D2D transmitter and its intended receiver (section 2). Equations (14) can be solved as linear programming optimization problem with the following, additional, constraint aiming to limit the effect of the D2D interference on the base station,

$$
d_{k, i}=d_{k, B} \varphi^{-1 / \alpha}
$$

Here, $d_{k, B}$ designates the spatial distance between the D2D transmitter and BS.

\section{Sinr-outage probability}

In this section, we choose outage probability as our metric for network performance evaluation. It signifies the complement of the probability of successful transmission resulting from the power and RB assignments being proposed. The rational for our choice is that, we envision the SINR outage as measuring the probability that the achieved transmission rate is falling below the desired communication rate, $R$, as a consequence of improper reuse-distances (lack of SINR). To see this, recall the maximum "Shannon" rate, $R$, wherein a one-to-one mapping between SINR and $R$ indicates that the outage probability can equivalently be stated in terms of the achieved transmission rate with, threshold-SINR $=2^{R}-1$.

More formally, the probability that the received SINR is below a given threshold $\varphi$, is defined as [20],

$$
\mathbb{P}(\operatorname{SINR} \leq \varphi)=\mathbb{P}\left(\mathrm{P}_{\mathrm{T}}^{\mathrm{k}} \mathrm{d}_{\mathrm{i}, \mathrm{k}}^{-\alpha} \leq \varphi\left(\sigma^{2}+\sum_{\mathrm{i}=1}^{\mathrm{n}} \sum_{\forall \mathrm{j} \neq \mathrm{i}} \mathrm{P}_{\mathrm{T}}^{\mathrm{i}} \mathrm{d}_{\mathrm{j}, \mathrm{i}}^{-\alpha}\right)\right)
$$

As mentioned earlier, when the desired signal $\mathrm{P}_{\mathrm{T}}^{\mathrm{k}} \mathrm{d}_{\mathrm{k}, \mathrm{i}}^{-\alpha}$ is subject to exponentially distributed Rayleigh fading, we obtain for the outage probability over link of a distance, $\mathrm{d}_{\mathrm{k}, \mathrm{i}}$, 


$$
\mathbb{P}(\operatorname{SINR} \leq \varphi)=1-\exp \left(\frac{-\varphi}{\mathrm{P}_{\mathrm{T}}^{\mathrm{k}}}\left(\sigma^{2}+\sum_{\mathrm{i}=1}^{\mathrm{n}} \sum_{\forall \mathrm{j} \neq \mathrm{i}} \mathrm{P}_{\mathrm{T}}^{\mathrm{i}} \mathrm{d}_{\mathrm{j}, \mathrm{i}}^{-\alpha}\right)\right),
$$

Where, $\mathrm{P}_{\mathrm{T}}^{\mathrm{k}}$ is the transmission power, the first term in the exponent of (16) depends on the (AWGN) noise power $\sigma^{2}$, while the second term depends on the interference (assumed Gaussian). According to equation (16), a message can be successfully decoded at the receiver if and only if the SINR at the receiver is greater than the threshold $\varphi$. If the SINR at the receiver does not exceed that threshold, the link experiences an outage (hence, denoted by SINR outage).

\section{Validation results}

Since interference is the main performance limiting factor in cellular networks. In this section, we restrict ourselves to presenting answers to such questions as how interference and, hence, outage probabilities are affected by the optimal transmission power and the resource allocation schemes being developed in this paper.

Consider a cellular system wherein a given cell serves certain number of cellular UEs, $K$ of which is selected to share their uplink resources with the cellular UEs in a D2D underlay fashion. We assume that the $(X, Y)$ coordinate of the users are known to both the UEs and the BS (section 2). Since D2D receivers suffer from the interference coming from cellular user's uplink transmission. To limit this interference, we use the power control scheme presented in section 4 hence, preventing cellular UEs from generating excess interference at the D2D receivers. On the other hand, D2D users share the same resources as cellular users in uplink; BS receives interference from D2D user's transmission. To reduce this interference, we use the concentric based resource allocation scheme presented in sections 3 .

In this respect, we have developed a simulation mode that realistically models the D2D underlay. The following summarizes the simulation parameters and their default values: Cell (subarea) level user distribution: Uniform, Distance attenuation: $L=35.3+37.6 * \log$ (d), Cell radius: $500 \mathrm{~m}$, UE thermal noise density: $-188 \mathrm{dBm} / \mathrm{Hz}$. Fig., (3) depicts the structure of the simulation model. In this model, a potential D2D UE receiving from a neighbor transmitter should be spatially separated from any other interferer by at least a distance $D$ known as the "reuse distance". Choosing $D$ generally depends on the acceptable SINR levels. In our study, we examine the effect of spatial separations between the D2D transmitters and the BS located at the central of cellular communications. The aim is to reduce the amount of interference induced at the BS receiver, hence, protecting the cellular communications. In this respect, we compare the proposed "concentric" based D2D RB assignment algorithm with the conventional (non- concentric) assignment.

At the beginning of the simulations, we assume the conventional, non-concentric scenario first. Here, the position of the BS is considered as point $(0,0)$ and the cellular UEs are having their positions fixed. Then, the D2D UEs are moved in steps of $20 \mathrm{~m}$ in both directions from the BS. For each position, based on the $(X, Y)$ coordinate estimation algorithm, we compute the transmission power (problem 13) for a given SINR target of 10 $d B$. Fig., (4) illustrates the SINR-outage probability at the BS. As can be seen when UEs are near the cell edge, the BS suffers less interference, hence, achieves less outage. 
On the other hand, since we are interested in studying the gains of having D2D communications integrated in cellular networks under the following constraints, $i$ ) concentric cell structure, ii) optimal transmission power control/interpolation, iii) quantized RB allocation scheme. Fig., (5) below, compares the outage probabilities obtained for the conventional and the optimized concentric scenarios. As can be seen, the results reveal the fact that the amount of interference induced at both the cellular and D2D UEs (C-UEs, D-UEs) and BS receivers depends not only on D2D spatial separation between D2D and the BS. Rather, it also depends on the transmission power optimization/interpolation as well as on the proper choice of the cellular RBs for sharing with the D2D candidates in an underlay fashion.



Fig. 4. Blocking probability at the BS: conventional scenario.

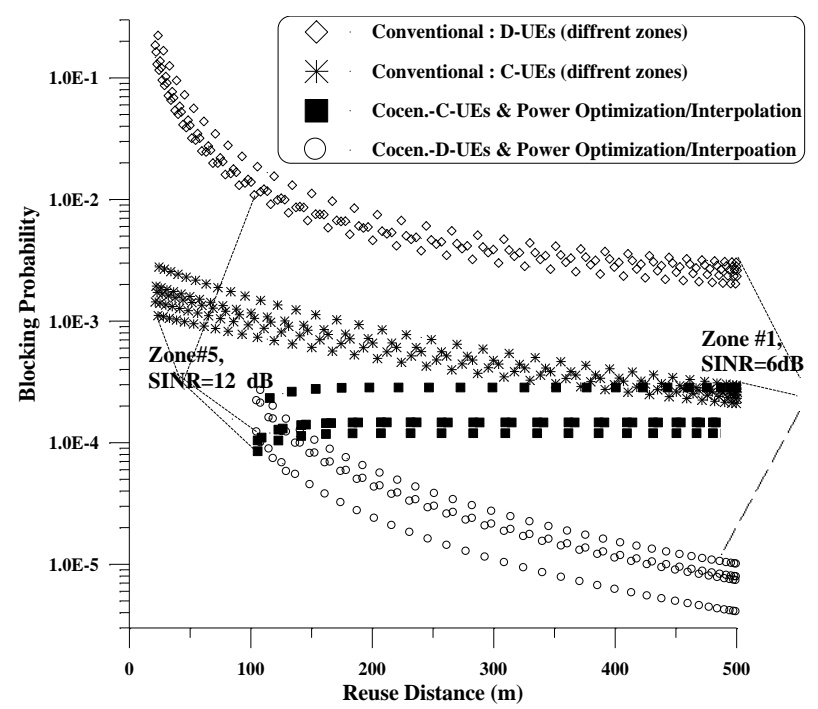

Fig. 5. Concentric cellular and D2D integration

Finally, the spatial throughput which is often conceived as indicating the wasted transmissions due to induced interference (wasted precious energy) is examined. Fig. (6), 
illustrates the achieved throughput as a result of the SINR-outage shown in Fig. (5). Let $\rho$ designate the achieved throughput and $\mathrm{R}$ the maximum transmission rate,

$$
\rho=\mathrm{R}(1-\mathbb{P}(\operatorname{SINR} \leq \varphi))
$$

As seen in Fig. (5), since our D2D approach outperformed that of the conventional schemes. It is reasonable to examine the achieved throughput of D2D communication based on our schemes only. As can be seen, as the distance between the two interfering links (BS and D2D UEs) is increased, the throughput increases due to the reduced amount of interference. However, due to physical limitations on the maximum UE's transmission power, increasing the distance between the D2D UE-pairs results in reduced throughput. With this in mind, network applications such as streaming media, high levels of outage would be unacceptable, and as such, it is desirable that the network operate in a low-outage scheme. We leave this observation for further investigations.

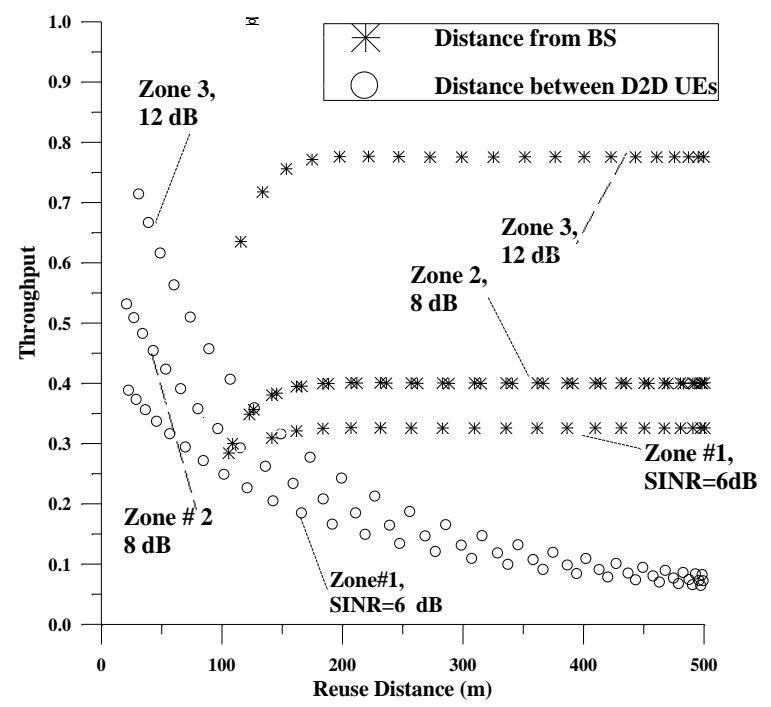

Fig. 6. D2D throughput in concentric cellular and D2D underlay

\section{Summary and conclusions}

In this paper, we have focused on the effect of the interference problem when D2D underlay share the channel with cellular networks. It is seen that the amount of interference depends not only on D2D transmit power but also on spatial distance between D2D transmitters and the cellular users. The solution approach we have presented in this paper is three folds: 1) a simple, practical approach for UE's $(X, Y)$ coordinate estimation in a typical cellular network scenario is presented. The aim is to enable efficient discovery and communications between cellular and proximate D2D users. 2) an optimal transmission power assignment/interpolation scheme is developed to set the transmission power of UEs located anywhere in the cell in order to compensate for the channel path loss as well as for the estimated noise and interference power induced at both the D2D and BS receivers. 3) a spatial orthogonal resource reuse scheme which can guarantee an average SINR level for the cellular and the D2D underlay is presented with some details. Finally, the SINR outage probability is considered as our metric for network performance evaluation. Simulation results have shown that using the concentric cellular cell structure outperforms the state of the art, conventional, assignment system. 
At this point, it is worth noticing that the approach presented in this paper can easily be implemented in real cellular networks without the need of new standardization work. In this respect, we consider our approach as simpler where the power control and resource allocations are separately considered with a significantly low complexity. For instance, in the first part of the paper, a soft resource allocation scheme which guarantees orthogonality among all RBs and, at the same time, guarantees maximum possible reuse distances is proposed. On the other hand, storing the channel states offline in a lookup table at the BS and broadcasting this data on less frequent basis is seen as further simplification compared with many schemes in the literature wherein non-convex objective functions are adopted and which had to be decomposed into (related) sub-problems, or solved using different approaches such as the Lagrange multipliers or iterative algorithms such as the sub-gradient algorithm.

\section{REFERENCES}

[1] METIS, "Mobile and wireless communications Enablers for the Twenty twenty Information Society, EU 7th Framework Program project, "Deliverable D1.1. Scenarios, requirements and KPIs for 5G, mobile and wireless system," Communications and Network, 2013, 5, 187 193

[2] K. Doppler, M. Rinne, C. Wijting, C. B. Riberio, and K. Hugl, "D2D communications underlaying an LTE cellular network," IEEE Communications Magazine, 7(12):42-49, December 2009.

[3] G. Fodor, E. Dahlman, S. Parkvall, G. Mildh, N. Reider, G. Miklos, and Z. Turanyi, "Design Aspects of Cellular Network Assisted Device-to-Device Communications," IEEE Communication Magazine, 50(3), 2012.

[4] M. Belleschi, G. Fodor, and A. Abrardo, "Performance Analysis of a Distributed Resource Allocation Scheme for D2D Communications," In IEEE Workshop on Machine-to-Machine Communications. IEEE, 2011.

[5] M.S. Corson, R. Laroia, J. Li, V. Park, T. Richardson, and G. Tsirtsis, "Toward proximityaware internetworking," IEEE Wireless Communications," 17(6):26-33, 2010.

[6] M. Belleschi, G. Fodor, D. Penda, M. Johansson†, A.Pradini, A. Abrardo, "Benchmarking Practical RRM Algorithms for D2D Communications in LTE Advanced," arXiv: 1306.5305v1 [cs.IT] 22 Jun 2013

[7] P. Jänis et al., "Interference-Aware Resource Allocation for Device-to-Device Radio Underlaying Cellular Networks,” IEEE VTC Spring, Barcelona, Spain, Apr. 2009.

[8] Marco Bellesch, G'abor Fodor, Demia Della Penda, Mikael Johansson, "Benchmarking Practical RRM Algorithms for D2D Communications in LTE Advanced," arXiv:1306.5305v1 [cs.IT] 22 Jun 2013

[9] A. Asadi, Q. Wang, and V. Mancuso, "A survey on device-to-device communication in cellular networks," available at: http://arxiv.org/abs/1310.0720.

[10] H. Min, J. Lee, S. Park, and D. Hong, "Capacity enhancement using an interference limited area for device-to-device uplink underlaying cellular networks," IEEE Transactions on Wireless Communication, vol. 10, no 12, pp. 3995-4000, 2011.

[11] J, Sun, M. Sheng, X. Wang, Y. Zhang, J. Liu, W. Junyu, "Resource allocation for maximizing the device-to-device communications underlaying LTE-Advanced networks," IEEE/CIC International Conference on Communications in China - Workshops (CIC/ICCC), pp. 60-64, August 2013.

[12] P. Phunchongharn, E. Hossain, and D. I. Kim, "Resource allocation for device-to-device communications underlaying LTE-advanced networks," IEEE Wireless Communications, vol. 20, no. 4, pp. 91-100, August 2013. 
[13] Jia Liu1, Bingbing Li1, Bing Lan1, Junren Chang, "A Resource Reuse Scheme of D2D Communication Underlaying LTE Network with Intercell Interference," arXiv:1405.2017v2 [cs.NI] 12 May 2014

[14] C.-H. Lee and M. Haenggi, "Interference and outage in Poisson cognitive networks," IEEE Transactions on Wireless Communications, vol. 11, pp. 1392-1401, April 2012.

[15] Li et al., Li, X. J., Seet, B.-C., and Chong, "Multihop cellular networks: Technology and economics," Computer. Networks., 52(2009):1825-1837.

[16] G. Mao et al., "Wireless sensor network localization techniques," Computer Networks 51 (2007) 2529-2553

[17] Raghothaman et al., B. Sternberg, G., Kaur, S., Pragada, R., Deng, T., and Vanganuru, "System architecture for a cellular network with cooperative mobile relay," In Vehicular Technology Conference (VTC Fall), 2011 IEEE, pages 1-5.

[18]N. Bulusu, J. Heidemann, and D. Estrin, "GPS-less low cost outdoor localization for very small devices," IEEE Personal Comm., 5(5):28-34, Oct. 2000.

[19] Burden, Richard L.; Faires, J. Douglas, Numerical Analysis. Belmont: Brooks/Cole(2004).

[20] Hesham ElSawy Q. Duong and O.-S. Shin, "Analytical Modeling of Mode Selection and Power Control for Underlay D2D Communication in Cellular Networks," arXiv:1405.2017v2 [cs.NI] 12 May 2014 
JES, Assiut University, Faculty of Engineering, Vol. 44, No. 1, January 2016, pp. 40 - 53

\section{الاتصالات المباشره بين وحدات الاتصالات الخلويه عن طريق الأتورئ

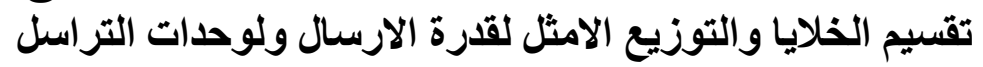

$$
\text { الملخص العربى: }
$$

يقدم هذا البحث نظاما جديدا للإتصالات المباشره بين وحدات الاتصالات الخلويه عن طريق تقيم الخلايا

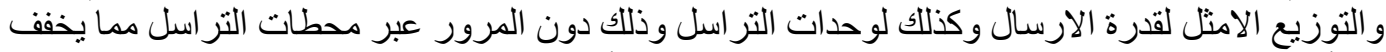
من أحمال الحركه بها وبالتالى زيادة قدرتها على خدمة عدد أكبر من المستخدمين. يقوم هذا النظام على التى

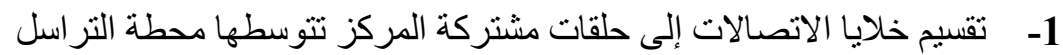

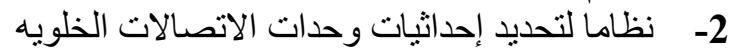

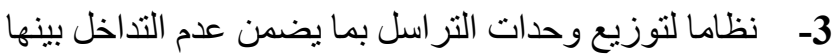

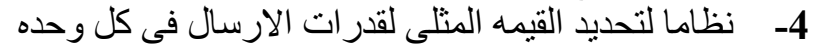

أظهرت النتائج كفاءة النظام المقدم مع بساطه تكوينه وذلك من خلال قدرته على التر اسل بسر عات أعلى

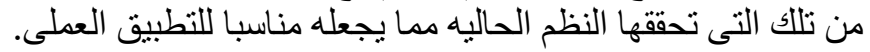

